\title{
GATA1 Expression in BCR/ABL1-negative Myeloproliferative Neoplasms
}

Naery Yang, M.D. ${ }^{1}$, Sholhui Park, M.D. ${ }^{1}$, Min-Sun Cho, M.D. ${ }^{2}$, Miae Lee, M.D. ${ }^{1}$, Ki-Sook Hong, M.D. ${ }^{1}$, Yeung Chul Mun, M.D. ${ }^{3}$, Chu-Myong Seong, M.D. ${ }^{3}$, Hee Jin Huh, M.D. ${ }^{4}$, and Jungwon Huh, M.D. ${ }^{1}$

Departments of Laboratory Medicine ${ }^{1}$, Pathology², and Internal Medicine ${ }^{3}$, College of Medicine, Ewha Womans University, Seoul; Department of Laboratory Medicine $^{4}$, Dongguk University Ilsan Hospital, Goyang, Korea

Background: This study aimed to determine GATA1 expression levels to better characterize subgroups in BCR/ABL1-negative myeloproliferative neoplasms (MPNs).

Methods: This study enrolled 49 patients diagnosed as having BCR/ABL1-negative MPN on the basis of the 2016 World Health Organization classification : nine polycythemia vera (PV), 17 essential thrombocythemia (ET), 12 prefibrotic primary myelofibrosis (prePMF), and 11 overt primary myelofibrosis (PMF). Relevant clinical and laboratory data were retrieved from the medical records. The molecular analysis of CALR and MPL mutations and quantification of JAK2 V617F allele burden were performed. GATA1 expression was assessed by an immunohistochemical assay on bone marrow biopsy. GATA1 expression was analyzed serially in 18 patients.

Results: GATA1 expression decreased significantly in PMF compared with that in other subtypes, while no statistical difference was identified between ET and prePMF. GATA1 expression did not differ according to the mutation profiles or the allele burden of JAK2 V617F, but it decreased significantly in patients with overt fibrosis or leukemic transformation.

Conclusions: Our results suggest that GATA1 expression is significantly low in PMF and decreases with progressive fibrosis and possibly with leukemic transformation, although our attempt to accurately distinguish between subgroups using GATA1 immunohistochemical approach did not achieve statistical significance. A large patient cohort with long term follow-up is required to evaluate the prognostic value of GATA1 expression.

Key Words: Polycythemia vera, Essential thrombocythemia, Prefibrotic primary myelofibrosis, Primary myelofibrosis, GATA1
Received: July 17, 2017

Revision received: September 19, 2017

Accepted: February 20, 2018

Corresponding author: Jungwon Huh Department of Laboratory Medicine, College of Medicine, Ewha Womans University, 1071 Anyangcheon-ro,

Yangcheon-gu, Seoul 07985, Korea

Tel: +82-2-2650-5320

Fax: +82-2-2650-5091

E-mail: JungWonH@ewha.ac.kr

Co-corresponding author: Hee Jin Huh Department of Laboratory Medicine, Dongguk University Ilsan Hospital, 27 Dongguk-ro, Ilsandong-gu, Goyang 10326, Korea

Tel: +82-31-961-7893

Fax: +82-31-961-7902

E-mail: hjhuh@duih.org

\begin{abstract}
@ Korean Society for Laboratory Medicine This is an Open Access article distributed under the terms of the Creative Commons Attribution Non-Commercial License (http://creativecommons.org/licenses/by-nc/4.0) which permits unrestricted non-commercial use, distribution, and reproduction in any medium, provided the original work is properly cited.
\end{abstract}

\section{INTRODUCTION}

BCR/ABL1-negative myeloproliferative neoplasms (MPNs) include polycythemia vera (PV), essential thrombocythemia (ET), prefibrotic primary myelofibrosis (prePMF), and overt primary myelofibrosis (PMF) [1, 2]. They are among the most frequent hematologic neoplasms, usually affecting the elderly population. Thrombosis, hemorrhage, evolution to myelofibrosis, and leukemic transformation represent the clinically relevant issues in the course of disease [3]. Especially in PMF, life expectancy was markedly lower than that in an age and sex-matched population in many studies $[4,5]$.

The 2016 WHO classification system divided PMF into prePMF and overt PMF on the basis of the absence or presence of grade $\geq 2$ bone marrow fibrosis [1].

The most commonly recognized mutation in BCR/ABL1-negative MPN is JAK2 V617F, followed by the CALR and MPL mutations $[6,7]$. There has been continued debate on how to sub- 
type BCR/ABL1-negative MPNs since none of the three major mutations are specific to any single subtype of MPN. Therefore, with this incomplete understanding of the molecular features of each subtype, an accurate characterization and standardization of morphological features is still being emphasized as a key to differentiate subtypes as per the 2016 WHO classification [1]. Furthermore, the disease subtypes often show considerable overlap in clinical and histopathological characteristics, especially in the early course of the disease. The differentiation between ET and prePMF has been particularly challenging, and diagnostic discrepancies exist even among experts $[8,9]$. The peripheral blood and bone marrow findings in patients with early or cellular phase PMF (prePMF) are similar to those of patients with ET. Hence, it is difficult yet imperative to discriminate between ET and prePMF as the clinical outcome in patients with prePMF is considerably worse than in those with ET [10-14].

Even though the 2016 WHO classification has provided criteria for the diagnosis of prePMF [1], bone marrow pathology provides major clues for the differential diagnosis of prePMF from ET. For both ET and prePMF, the histological hallmarks lie in the megakaryocyte (MK) population: ET is marked by proliferation of enlarged and mature MKs with deeply lobed and hypersegmented nuclei, whereas prePMF is characterized by proliferation of markedly atypical MKs that often form clusters. Megakaryocytic atypia involves maturation defects along with atypical nuclear features such as hypolobulation, hyperchromicity, irregular folding, and dense clustering [15]. However, the morphological evaluation of bone marrow specimens is subjective, and the reproducibility has been questioned [8, 9].

Therefore, we aimed to determine whether an immunohistochemical assay would allow for a more objective histopathological assessment of bone marrow MKs. Since each subtype of $B C R / A B L 1$-negative MPN is characterized by well-defined features during MK differentiation, we searched for immunohistochemical markers that could highlight abnormalities in MKs. GATA1, the founding member of the GATA family, is a DNA- and protein-binding transcription factor that is essential for erythroid precursor differentiation and MK maturation [16-20]. Precursor cells of MK lineage lacking GATA1 may arrest in maturation and undergo unrestrained proliferation [16-20]. Some studies reported that defective expression of GATA1 in MKs may be associated with myelofibrosis [16-20]. Moreover, the development of myelofibrosis has been documented in mice with reduced GATA1 gene expression [17, 19]. We investigated GATA1 expression at diagnosis and during follow-up for better characterization of each subgroup of $B C R / A B L 1$-negative MPNs.

\section{METHODS}

\section{Study and control groups}

To select study subjects, we re-evaluated bone marrow slides and reviewed clinical and laboratory data of patients with BCR/ ABL1-negative MPN, MPN, unclassifiable (MPN-U), or myelodysplastic syndrome/MPN, unclassifiable (MDS/MPN-U) between 1998 and 2015 at Ewha Womans University Mokdong Hospital, Seoul, Korea. On application of the 2016 WHO classification [1] after a thorough re-evaluation by two pathologists, 14 patients were reclassified from their original diagnoses, which were based on the 2001 or 2008 WHO classification [21, 22]. Five patients with ET, two with MPN-U, and three with MDS/ MPN-U were reclassified as prePMF, and three MPN-U and one MDS/MPN- $U$ were reclassified as PV.

Forty-nine patients who had BCR/ABL1-negative MPN (nine PV, 17 ET, 12 prePMF, and 11 PMF) according to the 2016 WHO classification were included in this study. Among 17 patients with $E T$, four had megakaryocytic atypia favoring the diagnosis of prePMF. In one of these four patients, MF-1 fibrosis was present, which was rare in ET. However, these four patients were finally diagnosed as having ET because the diagnostic criteria for prePMF were not met. This study was exempted from review by the Institutional Review Board of Ewha Womans University, and all information was de-identified.

The control group for GATA1 stain comprised 40 patients, of which nine had BCR/ABL1-positive chronic myeloid leukemia (CML), 16 had lymphoma without bone marrow involvement, and 15 had benign hematologic diseases (BHD) (eight idiopathic thrombocytopenic purpura, two iron deficiency anemia, and five normocellular marrow).

Electronic medical records were reviewed for retrospective collection of relevant clinical and laboratory data including the results of cytogenetics and JAK2 mutation. The JAK2 V617F mutation was identified in all patients with $\mathrm{PV}, 53 \%$ of those with ET, $67 \%$ of those with prePMF, and $40 \%$ of those with PMF. Leukemic transformation was observed in $8 \%$ patients with prePMF, whereas no patients with ET developed acute leukemia. Moreover, $67 \%$ patients with prePMF eventually developed fibrosis of $M F>2$ in the course of the disease over 19 to 82 months (median 55.5 months), whereas no patient with ET developed overt fibrosis. The baseline characteristics of study group are summarized in Supplemental Data Table S1.

The follow-up period varied from nine to 167 months (median 86.5 months). No patient received JAK2 inhibitor therapy or stem cell transplantation during the follow-up period. 


\section{Cytogenetics and molecular assays}

Cytogenetic analysis was performed by unstimulated 24- and/or 48-hour culture and conventional G-banding techniques using the fresh bone marrow aspirates obtained at diagnosis. For molecular assays, genomic DNA was extracted from fresh bone marrow aspirates or stored bone marrow cell pellets fixed for cytogenetic analysis using a DNA purification kit (Qiagen, Hilden, Germany). An allele-specific, real-time PCR assay (Real-Q JAK2 V617F kit, BioSewoom, Seoul, Korea) was used for qualitative analysis of the JAK2 V617F mutation.

Quantification of JAK2 V617F allele burden was performed in 25 patients with JAK2 mutation. Fifteen patients without JAK2 mutation were subjected to molecular analysis of $C A L R$ and $M P L$ mutations.

The allele burden of JAK2 V617F was measured as the percentage of mutant allele with respect to total JAK2 (mutant plus wild-type), using the JAK2 V617F quantification kit (BioSewoom). The CALR mutations were assessed by Sanger sequencing. Exon 9 of CALR was amplified using the following primers: forward, 5'-CAT TCA TCC TCC AGG TCA AG-3' and reverse, 5'-TGA AAG TTC TCG AGT CTC ACA G-3'. PCR was performed with the following cycle profile: an initial 5 minutes denaturation at $94^{\circ} \mathrm{C}$, 35 cycles of $94^{\circ} \mathrm{C}$ for 30 seconds, $60^{\circ} \mathrm{C}$ for 30 seconds, $72^{\circ} \mathrm{C}$ for 30 seconds, and a final 7 minutes extension at $72^{\circ} \mathrm{C}$.

The MPL W515L/K mutations were assessed by real-time PCR (Real-Q MPL W515L/K screening kit, BioSewoom), according to the manufacturer's instructions.

\section{Immunohistochemical assays of GATA1 expression}

The archived formalin-fixed and paraffin-embedded bone marrow biopsies were used for GATA1 immunohistochemical stain. Bone marrow biopsy samples obtained at diagnosis from 49 pa-
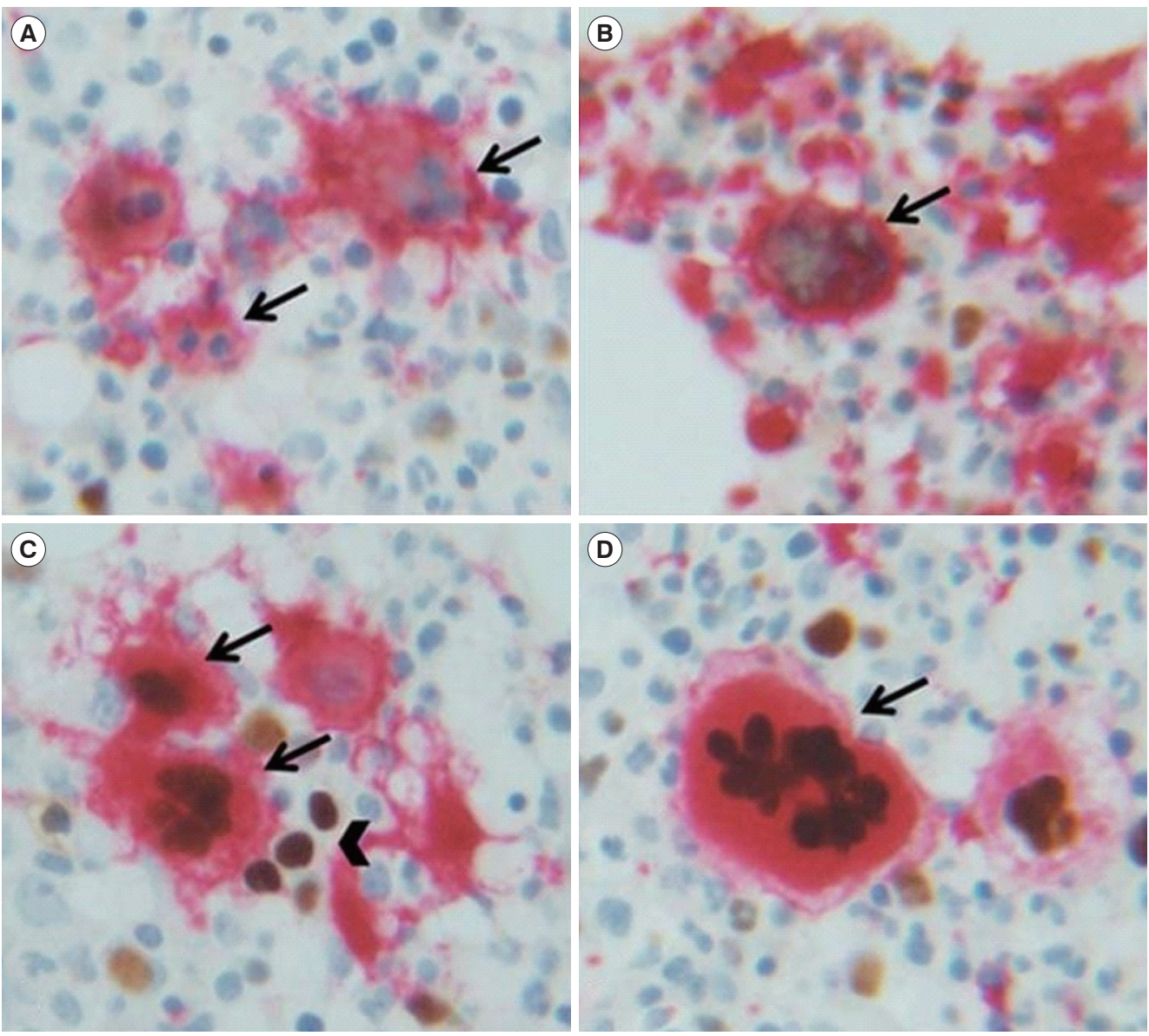

Fig. 1. Representative images of GATA1 immunoexpression in bone marrow biopsy samples according to the intensity of the reaction $(\times 400)$ : (A) negative, (B) weak, (C) moderate, and (D) strong. Megakaryocytes (arrows) are identified by CD61-stained (pink-colored) cytoplasm. GATA1-stained nuclei of megakaryocytes colored light to dark brown depending on the immunointensity. GATA1-stained nuclei are also seen in erythroid cells (arrowhead) without the CD61-positive cytoplasm. 
tients with $B C R / A B L 1$-negative MPN were stained and analyzed, and serial analyses were available for 18 patients. To better identify MKs, dual staining with GATA1 and CD61 was conducted. The immunohistochemical staining of GATA1 was performed using GATA1 monoclonal antibodies (GATA1 [D52H6] XP rabbit monoclonal antibodies, Cell Signaling Technology, Danvers, MA, USA), and the cytoplasm of MKs were stained with CD61 mouse monoclonal antibodies (EMD Millipore Co., Temecula, CA, USA). Antibodies were used at 1:100 dilutions. All MKs (median, 72; range, 10-345 MKs per slide for one patient) were evaluated at $400 \times$ magnification. Bone marrow specimens with less than 10 MKs were excluded from the GATA1 immunohistochemical assay analysis. MKs were classified as GATA1-positive, if the nuclei were clearly GATA1-positive (brown) with CD61-staining (red) in the cytoplasm.

GATA1 expression was semi-quantitatively evaluated in terms of GATA1 score, which was obtained by multiplying the intensity score by the positive cell percentage score [23, 24]. GATA1 immunostaining intensity was rated on a scale from 0 to 3: negative, 0; weak, 1; moderate, 2; and strong, 3 (Fig. 1). The percentage of positive MKs (the number of GATA1-stained MKs $\times 100$ / total number of MKs) was graded from 0 to 4: less than $1 \%, 0$; 1-20\%, 1; 21-50\%, 2; 50-80\%, 3; and 81-100\%, 4.

\section{Statistical analysis}

The differences in continuous variables (GATA1 score, age, hemoglobin level, white blood cell count, platelet count, lactate dehydrogenase, and JAK2 V617F allele burden) between more than three groups were evaluated using the Kruskal-Wallis test and subsequently calculated with post hoc Mann-Whitney test between each two groups. When the independent variables were fewer than three, the Mann-Whitney test was used. Categorical variables (sex; presence of leukoerythroblastosis, splenomegaly, myelofibrosis, cytogenetic abnormalities, or any specific mutation; progression to acute leukemia; and development of fibrosis) were compared between groups using chi-square tests, and subsequent $P$ values were calculated using Fisher's exact test.

All statistical analyses were performed with MedCalc for Windows, version 16.4.3 (MedCalc Software, Ostend, Belgium). P values $<0.05$ were considered statistically significant.

\section{RESULTS}

\section{Molecular analyses}

The CALR mutation was detected in 18\% patients with ET, 22\% patients with prePMF, and $30 \%$ patients with PMF. Furthermore,
$29 \%$ patients with ET, $11 \%$ patients with prePMF, and 30\% patients with PMF were negative for all three mutations (JAK2, $C A L R$, and $M P L$ ). There were no differences in the frequency of $C A L R$ or triple negative mutations among ET, prePMF, and PMF.

The median value and the range of the allele burden of JAK2 V617F at initial diagnosis for each subtype of BCR/ABL1-negative MPN are presented in Supplemental Data Table S1. The allele burden of JAK2 V617F differed significantly between the groups ( $P=0.022)$, and subsequent post hoc comparisons verified that it was lower in ET than in PV $(P<0.001)$, prePMF ( $P=$ $0.022)$, or PMF $(P=0.033)$. No significant difference in the JAK2 V617F allele burden was observed between the disease groups other than ET.

JAK2 V617F allele burdens of more than $50 \%$ were observed in $89 \%$ patients with $\mathrm{PV}, 50 \%$ patients with prePMF, and $67 \%$ patients with PMF, but none was observed in patients with ET.

\section{GATA1 expression analysis}

1) Comparison of GATA1 expression according to disease group The GATA1 score differed significantly between disease groups $(P=0.003)$. The cut-off score for normal GATA1 expression was set at 6 based on the finding that no patient in the BHD group showed a score less than 6 . A GATA1 score less than 6 was considered as low GATA1 expression.

PV and prePMF showed a GATA1 score distribution similar to that of BHD. For PMF, the GATA1 score was significantly lower than that of PV, prePMF, or BHD. Further, 73\% patients with PMF scored less than 6 , whereas no patients had a GATA1 score less than 6 in the PV or prePMF groups (Fig. 2).

Patients with ET showed a heterogeneous GATA1 score distribution. Features that favored the diagnosis of prePMF, such as increased lactate dehydrogenase and/or megakaryocytic atypia, were present in four patients with ET who scored less than 6 at diagnosis. MF-1 fibrosis was present in one of these four patients, which was rare in ET. However, they were classified as ET because the patients either had normocellular marrow or the minor criterion for prePMF was not met. No statistically significant difference in GATA1 score was found between the ET and prePMF groups. GATA1 expression in the CML and lymphoma without bone marrow involvement groups was heterogeneous, as shown in Fig. 2.

2) Comparison of GATA1 expression with respect to myelofibrosis grade, mutation profile, or JAK2 V617F allele burden

GATA1 expression was compared between two groups in the $B C R / A B L 1$-negative MPNs with specimens obtained at diagno- 


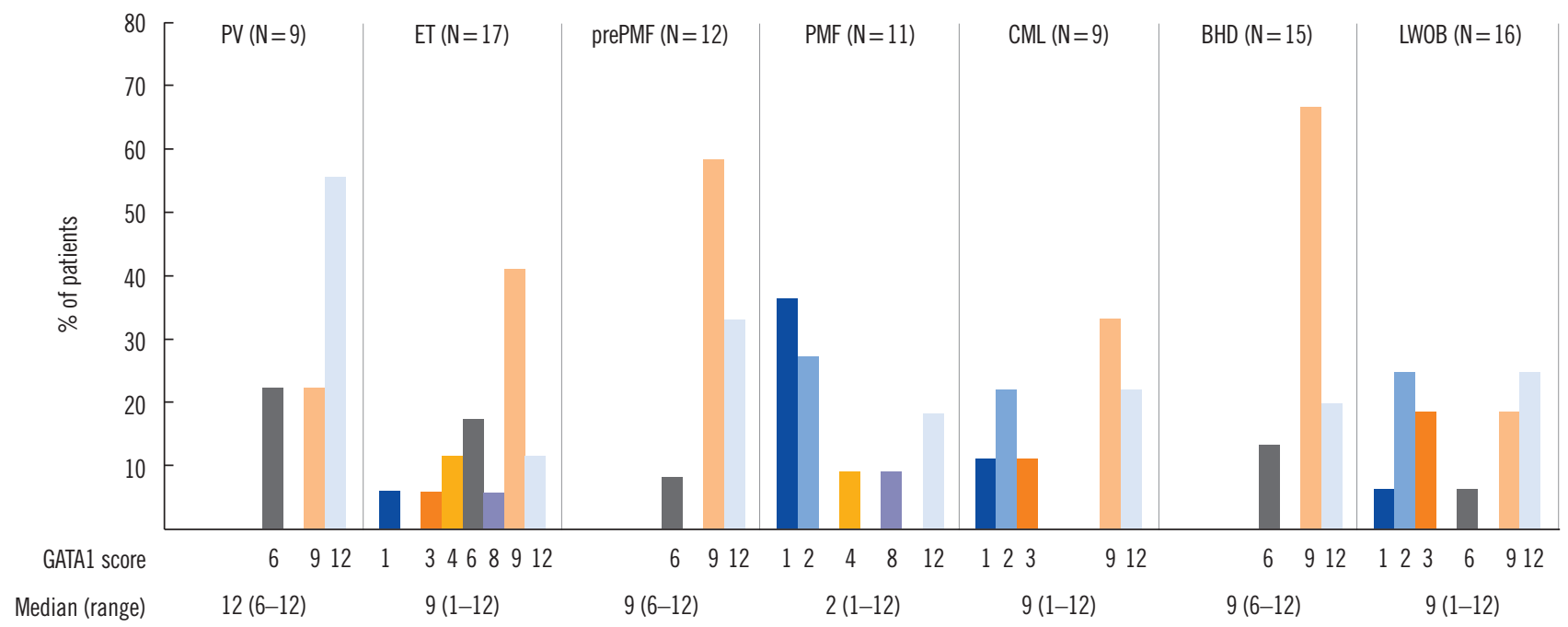

Fig. 2. Distribution of GATA1 expression according to disease groups $(P=0.004)$.

Abbreviations: BHD, benign hematologic disease; CML, chronic myeloid leukemia; ET, essential thrombocythemia; LWOB, lymphoma without bone marrow involvement; PMF, primary myelofibrosis; prePMF, prefibrotic primary myelofibrosis; PV, polycythemia vera.

sis: one with $\mathrm{WHO}$ myelofibrosis grade of $\mathrm{MF} \geq 2(\mathrm{MF}-2$ or 3$)$ and the other with $\mathrm{MF}<2$ (MF-0 or 1 ). The median value of GATA1 score in the $M F \geq 2$ group was significantly lower than in the $\mathrm{MF}<2$ group $(P=0.002)$. GATA1 expression according to myelofibrosis grade $(M F \geq 2$ vs $M F<2$ ) at the first follow-up yielded results similar to those obtained at diagnosis $(P=0.012)$ (Table 1$)$.

When GATA1 scores were compared according to mutation profile (JAK2, CALR, and triple negative mutations) at diagnosis, the difference between groups was not significant $(P=0.436)$, although the median value of the GATA1 score was lowest in the triple negative group (Table 1 ).

The median value of the GATA1 score tended to increase as JAK2 V617F allele burden increased, but no statistically significant difference was observed $(P=0.428)$ (Table 1 ).

In the PV, ET, and prePMF groups, no patient had both JAK2 V617F burden $>50 \%$ and GATA1 score $<6$ at diagnosis, whereas $33 \%$ patients with PMF showed both traits, as shown in Supplemental Data Table S2.

3) Serial analysis of GATA1 expression and clinical course

Figs. 3 and 4 depict GATA1 expression and clinical events in patients with follow-up bone marrow biopsies. In patients with PV, the GATA1 score on diagnosis was 9 or 12 and remained higher than 6 throughout the disease course until the development of overt myelofibrosis ( $M F \geq 2$ ). GATA1 expression decreased to scores of 1-3 with progression of myelofibrosis, except for one patient (patient PV-4).

Among the five patients with ET, three (patients ET-4, ET-8,
Table 1. Relationship of GATA1 expression in patients with $B C R$ / $A B L 1$-negative MPN with myelofibrosis grade, mutation profiles, and JAK2 V617F allele burden

\begin{tabular}{|c|c|c|}
\hline Variable & $\begin{array}{l}\text { GATA1 score } \\
\text { Median (range) }\end{array}$ & $p^{*}$ \\
\hline Myelofibrosis grade ${ }^{\dagger}$ at diagnosis $(\mathrm{N}=49$ ) & & 0.002 \\
\hline$M F<2(N=38)$ & $9(1-12)$ & \\
\hline$M F \geq 2(N=11)$ & $2(1-12)$ & \\
\hline Myelofibrosis grade ${ }^{\dagger}$ at the first follow-up $(\mathrm{N}=18)$ & & 0.012 \\
\hline$M F<2(N=14)$ & $9(2-12)$ & \\
\hline$M F \geq 2(N=4)$ & $2(1-9)$ & \\
\hline Mutation at diagnosis $(\mathrm{N}=35)$ & & 0.436 \\
\hline JAK2 V617F $(\mathrm{N}=28)$ & $9(1-12)$ & \\
\hline $\operatorname{CALR}(\mathrm{N}=8)$ & $9(1-12)$ & \\
\hline$M P L(\mathrm{~N}=0)$ & $N / A$ & \\
\hline Triple negative $(\mathrm{N}=9)$ & $6(1-12)$ & \\
\hline JAK2 V617F allele burden at diagnosis $(\mathrm{N}=25)$ & & 0.428 \\
\hline $1-20 \%(N=1)$ & $6(6)$ & \\
\hline $21-50 \%(N=10)$ & $12(3-12)$ & \\
\hline $51-80 \%(N=8)$ & $12(6-12)$ & \\
\hline $80-100 \%(N=6)$ & $12(1-12)$ & \\
\hline
\end{tabular}

*Significant values are in boldface; ${ }^{\dagger} \mathrm{WHO} 2016$ myelofibrosis grading system was adopted [1].

Abbreviations: MF, myelofibrosis; N/A, not applicable.

and ET-13) showed a steadily high GATA1 score (6 or higher). Two patients (ET-15 and ET-16) showed low GATA1 expression at diagnosis with a score of 4 , but the scores increased to 6 or 

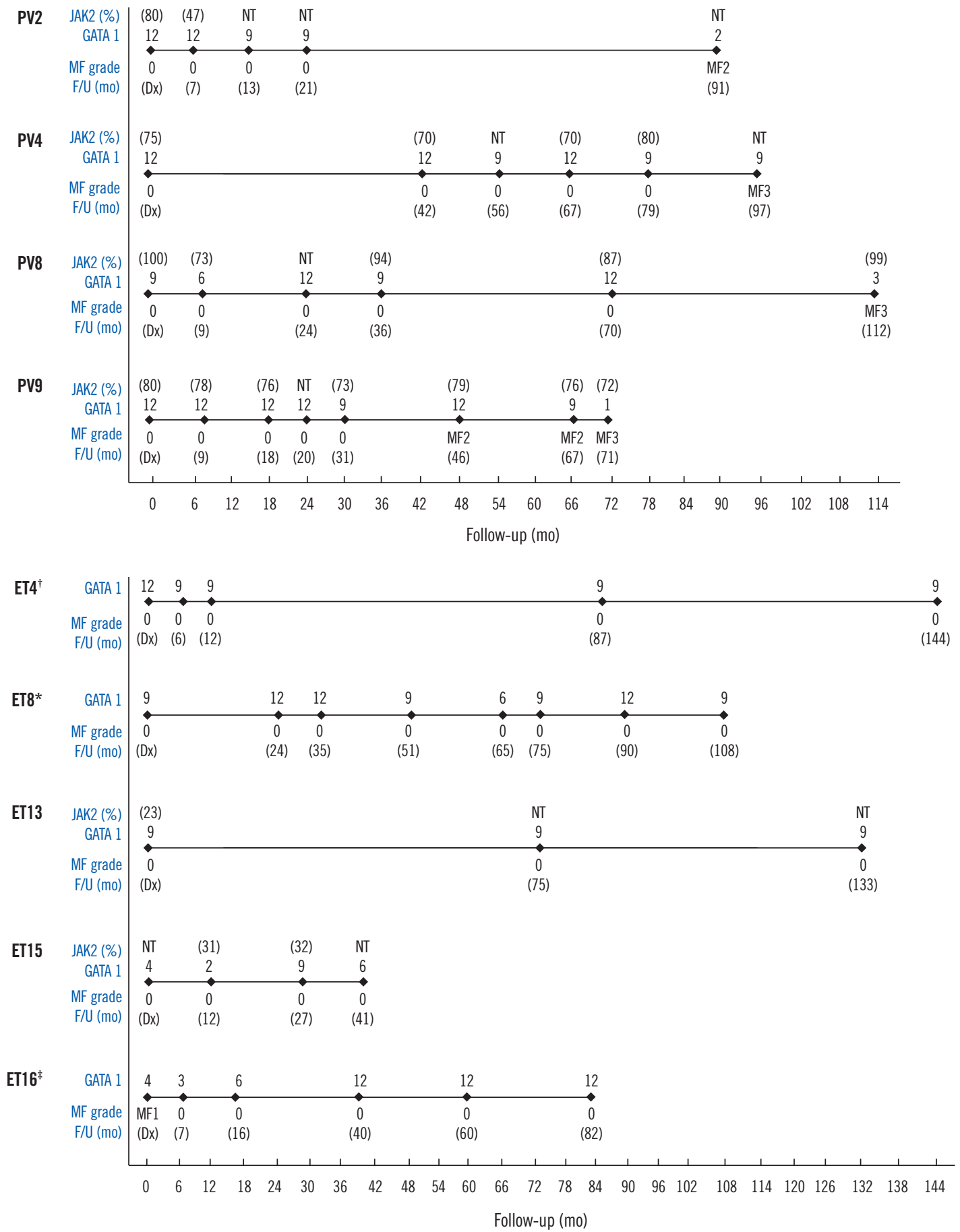

Fig. 3. Serial analysis of GATA1 expression, the allele burden of JAK2 V617F, and clinical events in patients with polycythemia vera or essential thrombocythemia.

${ }^{*}$ CALR type 1 mutated; ${ }^{\dagger} C A L R$ type 2 mutated; ${ }^{\dagger}$ Triple negative.

Abbreviations: ET, essential thrombocythemia; F/U, follow-up; mo, month; MF, myelofibrosis; NT, not tested; PV, polycythemia vera.

higher in the follow-up course. Patient ET-16 initially showed

MF-1 myelofibrosis and low GATA1 score, but the fibrosis was reversed during the follow-up course along with increase in GATA1 score.
The GATA1 score decreased from 9 to 4 when the disease transformed to acute myeloid leukemia for one patient (prePMF-1). The GATA1 score of another patient (prePMF-2) decreased from 9 to 4 and the blasts increased in number (17\% in bone mar- 

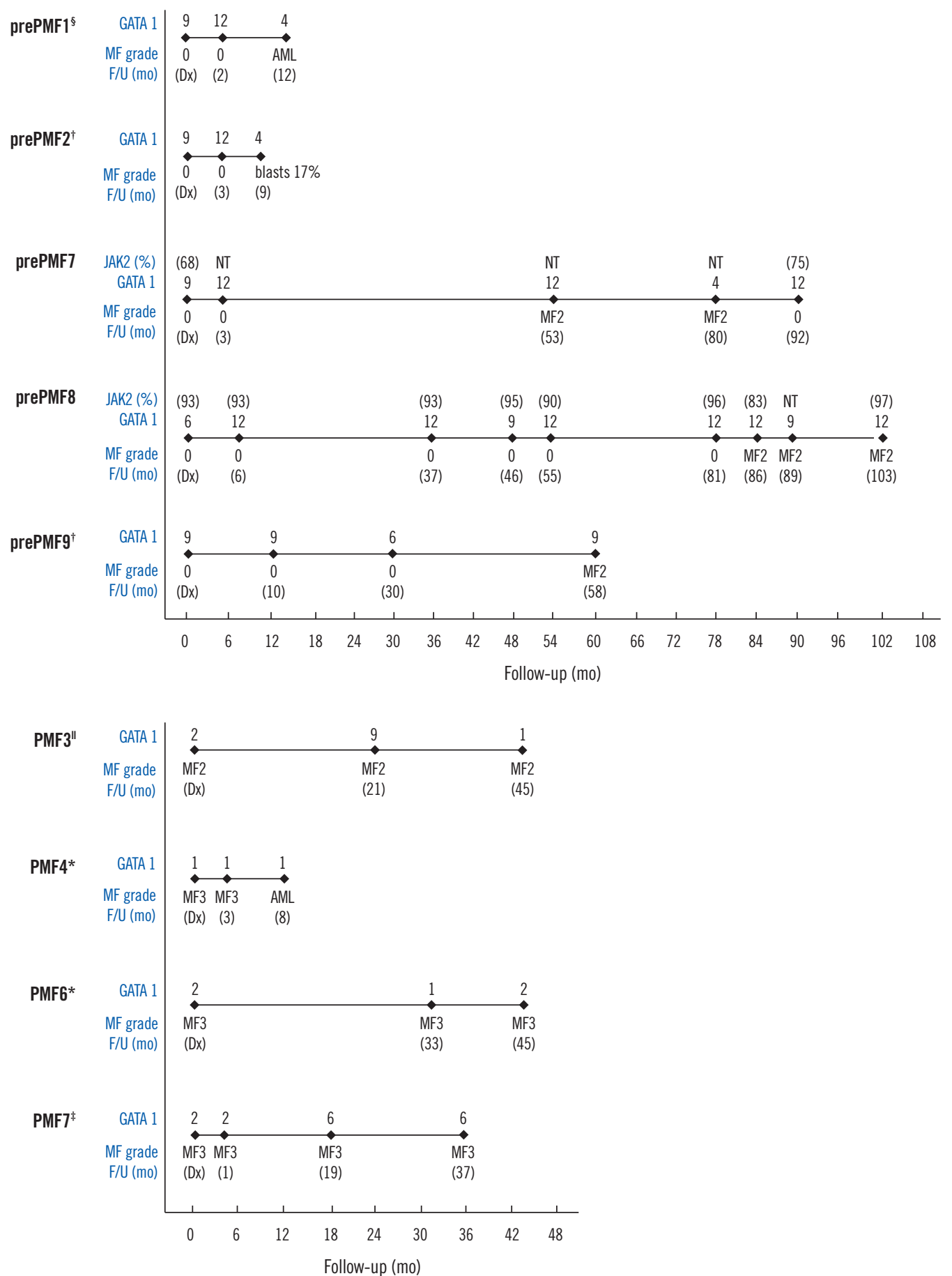

Fig. 4. Serial analysis of GATA1 expression, the allele burden of JAK2 V617F, and clinical events in patients with prefibrotic primary myelofibrosis or primary myelofibrosis.

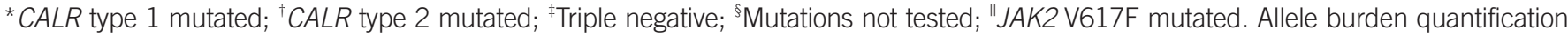
was not performed.

Abbreviations: AML, transformation to acute myeloid leukemia; F/U, follow-up; mo, month; MF, myelofibrosis; NT, not tested; PMF, primary myelofibrosis; prePMF, prefibrotic primary myelofibrosis.

row differential counts). However, in three patients with prePMF (prePMF-7, prePMF-8, and prePMF-9), the GATA1 scores re- mained high (score of 9-12) even when the myelofibrosis developed to MF-2. 
In patients with PMF (PMF-4 and PMF-6), the GATA1 score was 1 or 2 at diagnosis, which remained unchanged during follow-up with leukemic transformation or extensive fibrosis. The GATA1 score of two patients (PMF-3 and PMF-7) was 2 at diagnosis, which increased during follow-up without improvement of fibrosis on bone marrow biopsy.

JAK2 V617F allele burdens did not show significant changes during follow-up in patients with PV, ET, or prePMF although GATA1 expression changed with development of myelofibrosis or leukemic transformation.

\section{DISCUSSION}

We demonstrated that GATA1 expression was significantly lower in PMF than in other subtypes and the allele burden of JAK2 V617F was significantly lower in ET than in other subtypes. GATA1 expression did not differ according to the mutation profiles, but significantly decreased in patients with progressed fibrosis or leukemic transformation.

The frequencies of JAK2 V617F and CALR mutations in PV, ET, and PMF were similar to those found in previous studies [6, 7, 25-27]. Previous studies reported significantly higher allele burdens of JAK2 V617F in PV and PMF than in ET [27-35]. We also demonstrated that the burden of the JAK2 V617F allele in patients with ET was significantly lower than in patients with PV or PMF. The allele burden of JAK2 V617F was significantly higher $(P=0.022)$ in the prePMF group (median, 50.4; range, 31.693.1) than in the ET group (median, 29.8; range, 19.7-50.0). A JAK2 V617F allele burden higher than 50\% was not detected in any patient with ET, similar to the findings of previous studies [10, 28-30, 32]. Our results supported that a diagnosis of ET was less likely when a patient's JAK2 V617F allele burden was high (more than 50\%) [28-30, 34].

We assessed GATA1 expression in MKs to demonstrate its clinical relevance in distinguishing between $B C R / A B L 1$-negative MPN subtypes, especially prePMF from ET. GATA1 is a transcription factor that regulates maturation and differentiation of erythroid cells and MKs; Owing to genetic alterations, GATA1-low mice are both anemic and thrombocytopenic at birth [16, 36]. Few studies have shown that the number of MKs with retarded nuclear and cytoplasmic development increased significantly in GATA1-low mice [16, 17, 36]. Overall, impaired control of both MK proliferation and maturation is conceivably the result of low GATA1 expression. An animal model also showed that mice with defective GATA1 expression eventually developed myelofibrosis [19]. Other studies have also demonstrated decreased GATA1 expression in patients with PMF [17, 20]. In agreement with these previous studies, we showed that GATA1 expression was reduced in patients with overt myelofibrosis and that more than $60 \%$ patients with PMF had low GATA1 expression. GATA1 score less than 6 was observed in 24\% (four out of 17) patients with ET, including patients ET-15 and ET-16 with features which favored the diagnosis of prePMF; however, these patients did not fulfill the complete diagnostic criteria for prePMF. We speculated that these patients were in the gray area of diagnosis where it was uncertain whether they truly belonged to the ET or prePMF. Indeed, few studies have shown borderline ET/prePMF in 5-18\% patients with ET [37, 38].

In the serial analysis, GATA1 expression was also low in patients with PV who developed post-PV myelofibrosis during followup. The GATA1 score was significantly lower in the BCR/ABL1negative patients with MPN and myelofibrosis of $M F \geq 2$ than in those with $M F<2$, irrespective of the disease subtype. Two patients with PMF showed unexpected GATA1 score-fluctuation during the follow up course, which warrant further research in future. Interestingly, two patients with prePMF showed progressive decrease in GATA1 expression during leukemic transformation. Since the somatic mutation of GATA1 that reduces GATA1 expression is involved in the accumulation of immature erythroid and MKs progenitor cells [39, 40], these cells may subsequently acquire additional genetic hits and contribute to multi-step leukemogenesis [39, 40]. The possible association between reduced GATA1 expression and leukemogenesis should be investigated in the future.

Our study has some limitations. The small patient population for each subtype of $B C R / A B L 1$-negative MPN and the retrospective design of the study did not allow us to draw a definitive conclusion. Since an accurate histological diagnosis is still being emphasized in the 2016 WHO classification, further research on immunohistochemical markers is warranted for better and more standardized morphological evaluation.

In conclusion, although our attempt to accurately distinguish between subgroups using a GATA1 immunohistochemical approach did not achieve statistical significance, our results suggest that GATA1 expression decreases with progressive fibrosis and possibly with leukemic transformation.

\section{Authors' Disclosures of Potential Conflicts of Interest}

No potential conflicts of interest relevant to this article were reported. 


\section{Acknowledgment}

This study was supported by the Basic Science Research Program of the National Research Foundation of Korea, funded by the Ministry of Education, Science, and Technology (NRF-2012R1A1A2044138), and the Ewha Womans University Scholarship of 2015.

\section{REFERENCES}

1. Arber DA, Orazi A, Hasserjian R, Thiele J, Borowitz MJ, Le Beau MM, et al. The 2016 revision to the World Health Organization classification of myeloid neoplasms and acute leukemia. Blood 2016;127:2391-405.

2. Levine RL. Another piece of the myeloproliferative neoplasms puzzle. $\mathrm{N}$ Engl J Med 2013;369:2451-2.

3. Vannucchi AM, Guglielmelli P, Tefferi A. Advances in understanding and management of myeloproliferative neoplasms. CA Cancer J Clin 2009; 59:171-91.

4. Cervantes F, Passamonti F, Barosi G. Life expectancy and prognostic factors in the classic $B C R / A B L$-negative myeloproliferative disorders. Leukemia 2008;22:905-14.

5. Tefferi A, Huang J, Schwager S, Li CY, Wu W, Pardanani A, et al. Validation and comparison of contemporary prognostic models in primary myelofibrosis: analysis based on 334 patients from a single institution. Cancer 2007; 109:2083-8.

6. Tefferi A, Lasho TL, Finke CM, Knudson RA, Ketterling R, Hanson CH, et al. CALR vs JAK2 vs MPL-mutated or triple-negative myelofibrosis: clinical, cytogenetic and molecular comparisons. Leukemia 2014;28: 1472-7.

7. Tefferi A and Pardanani A. Myeloproliferative neoplasms: A contemporary review. JAMA Oncol 2015;1:97-105.

8. Barbui T, Thiele J, Vannucchi AM, Tefferi A. Problems and pitfalls regarding WHO-defined diagnosis of early/prefibrotic primary myelofibrosis versus essential thrombocythemia. Leukemia 2013;27:1953-8.

9. Buhr T, Hebeda K, Kaloutsi V, Porwit A, Van der Walt J, Kreipe H. European Bone Marrow Working Group trial on reproducibility of World Health Organization criteria to discriminate essential thrombocythemia from prefibrotic primary myelofibrosis. Haematologica 2012;97:360-5.

10. Barosi G. Essential thrombocythemia vs. early/prefibrotic myelofibrosis: why does it matter. Best Pract Res Clin Haematol 2014;27:129-40.

11. Gisslinger H, Jeryczynski G, Gisslinger B, Wolfler A, Burgstaller S, Buxhofer-Ausch $\mathrm{V}$, et al. Clinical impact of bone marrow morphology for the diagnosis of essential thrombocythemia: comparison between the BCSH and the WHO criteria. Leukemia 2016;30:1126-32.

12. Barbui T, Thiele J, Passamonti F, Rumi E, Boveri E, Ruggeri M, et al. Survival and disease progression in essential thrombocythemia are significantly influenced by accurate morphologic diagnosis: an international study. J Clin Oncol 2011;29:3179-84.

13. Bjorkholm M, Hultcrantz M, Derolf AR. Leukemic transformation in myeloproliferative neoplasms: therapy-related or unrelated? Best Pract Res Clin Haematol 2014;27:141-53.

14. Thiele J, Kvasnicka HM, Mullauer L, Buxhofer-Ausch V, Gisslinger B, Gisslinger $\mathrm{H}$. Essential thrombocythemia versus early primary myelofibrosis: a multicenter study to validate the WHO classification. Blood 2011;117:5710-8.

15. Barbui T, Thiele J, Vannucchi AM, Tefferi A. Rationale for revision and proposed changes of the WHO diagnostic criteria for polycythemia vera, essential thrombocythemia and primary myelofibrosis. Blood Cancer J 2015;5:e337.

16. Shimizu R and Yamamoto M. GATA-related hematologic disorders. Exp Hematol 2016;44:696-705.

17. Vannucchi AM, Pancrazzi A, Guglielmelli P, Di Lollo S, Bogani C, Baroni $\mathrm{G}$, et al. Abnormalities of GATA-1 in megakaryocytes from patients with idiopathic myelofibrosis. Am J Pathol 2005;167:849-58.

18. Bresnick EH, Katsumura KR, Lee HY, Johnson KD, Perkins AS. Master regulatory GATA transcription factors: mechanistic principles and emerging links to hematologic malignancies. Nucleic Acids Res 2012;40:581931.

19. Garimella R, Kacena MA, Tague SE, Wang J, Horowitz MC, Anderson $\mathrm{HC}$. Expression of bone morphogenetic proteins and their receptors in the bone marrow megakaryocytes of GATA-1 (low) mice: a possible role in osteosclerosis. J Histochem Cytochem 2007;55:745-52.

20. Rinaldi CR, Martinelli V, Rinaldi P, Ciancia R, del Vecchio L. GATA1 is overexpressed in patients with essential thrombocythemia and polycythemia vera but not in patients with primary myelofibrosis or chronic myelogenous leukemia. Leuk Lymphoma 2008;49:1416-9.

21. Jaffe ES and World Health Organization. Pathology and genetics of tumours of haematopoietic and lymphoid tissues. 1st ed. Lyon and Oxford: IARC Press; Oxford University Press (distributor), 2001.

22. Swerdlow SH, International Agency for Research on Cancer, World Health Organization. WHO classification of tumours of haematopoietic and lymphoid tissues. 4th ed. Lyon: International Agency for Research on Cancer, 2008.

23. Ponce CC, de Lourdes FCM, Ihara SS, Silva MR. The relationship of the active and latent forms of TGF-beta1 with marrow fibrosis in essential thrombocythemia and primary myelofibrosis. Med Oncol 2012;29:233744.

24. Krajewska M, Krajewski S, Epstein JI, Shabaik A, Sauvageot J, Song K, et al. Immunohistochemical analysis of bcl-2, bax, bcl-X, and mcl-1 expression in prostate cancers. Am J Pathol 1996;148:1567-76.

25. Klampfl T, Gisslinger H, Harutyunyan AS, Nivarthi H, Rumi E, Milosevic JD, et al. Somatic mutations of calreticulin in myeloproliferative neoplasms. N Engl J Med 2013;369:2379-90.

26. Rotunno G, Mannarelli C, Guglielmelli P, Pacilli A, Pancrazzi A, Pieri L, et al. Impact of calreticulin mutations on clinical and hematological phenotype and outcome in essential thrombocythemia. Blood 2014;123: 1552-5.

27. Palandri F, Latagliata R, Polverelli N, Tieghi A, Crugnola M, Martino B, et al. Mutations and long-term outcome of 217 young patients with essential thrombocythemia or early primary myelofibrosis. Leukemia 2015; 29:1344-9.

28. Edahiro Y, Morishita S, Takahashi K, Hironaka Y, Yahata Y, Sunami Y, et al. JAK2V617F mutation status and allele burden in classical Ph-negative myeloproliferative neoplasms in Japan. Int J Hematol 2014;99:62534.

29. Hussein K, Bock O, Theophile K, von Neuhoff N, Buhr T, Schlue J, et al. JAK2(V617F) allele burden discriminates essential thrombocythemia from a subset of prefibrotic-stage primary myelofibrosis. Exp Hematol 2009;37:1186-93 e7.

30. Park SH, Chi HS, Cho YU, Jang S, Park CJ. The allele burden of JAK2V617F can aid in differential diagnosis of Philadelphia chromosomenegative myeloproliferative neoplasm. Blood Res 2013;48:128-32.

31. Shirane S, Araki M, Morishita S, Edahiro Y, Sunami Y, Hironaka Y, et al. Consequences of the JAK2V617F allele burden for the prediction of transformation into myelofibrosis from polycythemia vera and essential thrombocythemia. Int J Hematol 2015;101:148-53.

32. Yonal-Hindilerden I, Daglar-Aday A, Akadam-Teker B, Yilmaz C, Nalcaci 
M, Yavuz AS, et al. The burden of JAK2V617F mutated allele in Turkish patients with myeloproliferative neoplasms. J Clin Med Res 2015;7:16170.

33. Kiladjian JJ. The spectrum of JAK2-positive myeloproliferative neoplasms. Hematology Am Soc Hematol Educ Program 2012;2012:561-6.

34. Larsen TS, Pallisgaard N, Moller MB, Hasselbalch HC. The JAK2 V617F allele burden in essential thrombocythemia, polycythemia vera and primary myelofibrosis--impact on disease phenotype. Eur J Haematol 2007; 79:508-15.

35. Vannucchi AM, Antonioli E, Guglielmelli P, Pardanani A, Tefferi A. Clinical correlates of JAK2V617F presence or allele burden in myeloproliferative neoplasms: a critical reappraisal. Leukemia 2008;22:1299-307.

36. Vannucchi AM, Bianchi L, Paoletti F, Di Giacomo V, Migliaccio G, Migliaccio AR. Impaired GATA-1 expression and myelofibrosis in an animal model. Pathol Biol (Paris) 2004;52:275-9.

37. Brousseau M, Parot-Schinkel E, Moles MP, Boyer F, Hunault M, Rousselet MC. Practical application and clinical impact of the WHO histopathological criteria on bone marrow biopsy for the diagnosis of essential thrombocythemia versus prefibrotic primary myelofibrosis. Histopathology 2010;56:758-67.

38. Florena AM, Tripodo C, lannitto E, Porcasi R, Ingrao S, Franco V. Value of bone marrow biopsy in the diagnosis of essential thrombocythemia. Haematologica 2004;89:911-9.

39. Shimizu R and Yamamoto M. Contribution of GATA1 dysfunction to multistep leukemogenesis. Cancer Sci 2012;103:2039-44.

40. Shimizu R, Kobayashi E, Engel JD, Yamamoto M. Induction of hyperproliferative fetal megakaryopoiesis by an N-terminally truncated GATA1 mutant. Genes Cells 2009;14:1119-31. 